\section{Asociación entre polimorfismos del gen de adiponectina y estado nutricional en escolares de la comuna de Hualpén}

\author{
GLORIA ORELLANA ${ }^{1, \mathrm{a}}$, JORGE SAPUNAR ${ }^{4, \mathrm{~d}}$, \\ KATIA SÁEZ ${ }^{2, \mathrm{~b}}$, CLAUDIO AGUAYO ${ }^{1, \mathrm{c}}$, CARLOS CALVO ${ }^{1, \mathrm{c}}$, \\ CLAUDIA RADOJKOVIC ${ }^{1, \mathrm{c}}$, BENILDE RIFFO ${ }^{1, \mathrm{e}}$, ANDREA GLEISNER ${ }^{3}$, \\ SYLVIA ASENJO ${ }^{3}$, NATALIA ULLOA ${ }^{1, c, e}$

\section{Association between adiponectin gene polymorphisms and obesity in school age children from Hualpén, Chile}

Background: Several genetic polymorphisms of adiponectin have been associated to metabolic diseases as obesity and co-morbidities. Aim: To investigate if there are associations between $+45 T G,+276 G T,-11,377 C G y-11,391 G A$ adiponectin SNPs (single nucleotide polymorphism) with obesity in a Chilean children population. Material and Methods: A case-control study was performed in 241 obese and 126 normal weight children (7-11 years old) from the urban community of Hualpén, Biobio region. Children were classified as normal or obese, according to age and gender-specific percentiles defined by Center for Disease Control and Prevention (CDC). The analysis of serum markers was carried out using commercial kits. Adiponectin polymorphisms were determined through a High Resolution Melting (HRM)-enabled real time PCR and by DNA fragment sequencing. Results: The observed allelic frequencies of the studied SNPs were over 11\%. The 11,377CG polymorphism was associated with a high risk of obesity, calculated by the additive inheritance model (odds ratio $=1.389,95 \%$ confidence interval: $1.001-1.929, p=0.049)$. Conclusions: Obese school children of the Biobio Region, have an increased risk of carrying the susceptibility allele polymorphism 11377CG of adiponectin gene.

(Rev Med Chile 2012; 140: 1245-1252).

Key words: apM-1 protein; Child; Obesity; Polymorphisms, Single Nucleotide.

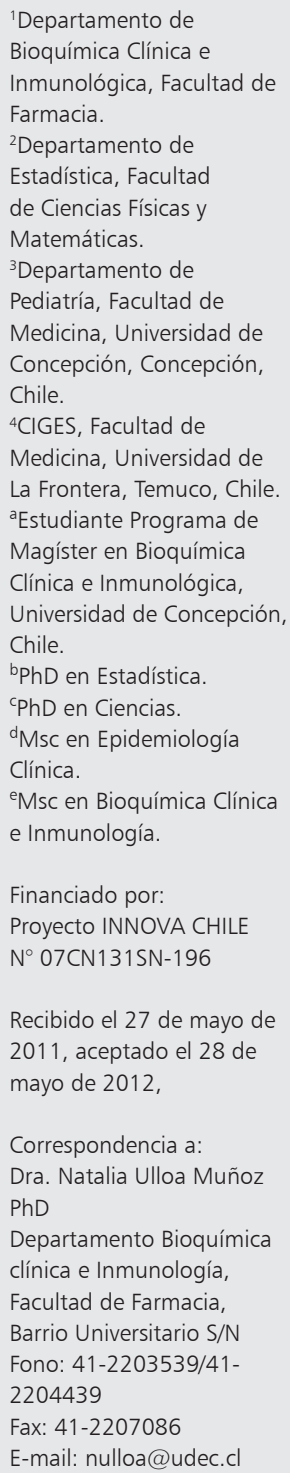

E 1 aumento en la prevalencia de la obesidad infanto-juvenil es uno de los problemas de salud pública más alarmante que enfrenta la humanidad $^{1,2}$. En Chile la prevalencia del exceso de peso (obesidad más sobrepeso) en niños menores de 6 años alcanzó 32,3\% en el año 2010 3,4.

Existen evidencias que demuestran que la obesidad aumenta el riesgo de padecer trastornos metabólicos como insulino-resistencia ${ }^{5,6}$, intolerancia a la glucosa ${ }^{2,5}$ y dislipidemias ${ }^{2,7}$. También se asocia con la aparición temprana de hiperten- sión ${ }^{2,7}$, diabetes mellitus 2 (DM2) $)^{2,4,5,7}$, eventos cardiovasculares ${ }^{2}$, hígado graso no alcohólico ${ }^{6}$, apnea obstructiva ${ }^{6}$ y asma . $^{7}$.

La adiponectina es una proteína producida por el adipocito durante su diferenciación ${ }^{4,8}$, que ejerce efectos metabólicos favorables tales como aumentar la sensibilidad a la insulina, inhibir la inflamación y la aterogénesis ${ }^{4,9,10}$. Estos efectos biológicos son mediados por la unión de esta adipoquina a dos tipos de receptores: ADIPOR1 presente en el músculo esquelético y cuya activación aumenta 
la captación de glucosa y la beta-oxidación de los ácidos grasos y ADIPOR2 presente en hígado y cuya activación reduce la gluconeogénesis ${ }^{10}$.

El gen de la adiponectina (AdipoQ) está localizado en el cromosoma 3q27. Estudios de barrido del genoma humano indican que éste corresponde a un locus de susceptibilidad para enfermedades cardiometabólicas. De esta forma se ha demostrado la asociación de polimorfismos genéticos de la adiponectina con obesidad, DM 2 y eventos cardiovasculares $^{11,12}$.

El polimorfismo +276GT (Intrón 2) se asocia con un aumento de los niveles de insulina plasmática, HOMA-IR y obesidad tanto en población adulta $^{11,13,16,17}$ como en población infantil ${ }^{18}$. El polimorfismo -11,377CG (Región promotora de AdipoQ) está asociado con aumento de la glicemia, insulinemia y disminución de la adiponectina plasmática en niños ${ }^{14,17,19} \mathrm{y}$ adultos ${ }^{13,20,21}$. El polimorfismo +45TG (Exón 2 de AdipoQ) se ha asociado con riesgo de insulino resistencia y obesidad en población adulta española, alemana, danesa y sueca ${ }^{11,13,15,16,21}$ y en población infantil se ha relacionado con mayor nivel de glicemia, insulina, HOMA-IR (homeostatic model assessmentinsulin) e hipo-adiponectinemia ${ }^{19}$. Finalmente, el polimorfismo -11,391GA ha sido asociado con mayor nivel de insulina plasmática y HOMA-IR en niños ${ }^{19} y$ con riesgo de DM2 en adultos ${ }^{17,22}$.

El objetivo de este estudio fue establecer si existe asociación entre la presencia de los polimorfismos +45TG (rs2241766), +276GT (rs15010299), -11.377CG (rs266729), -11,391GA (rs17300539) y el estado nutricional (Obeso/no obeso) de escolares pertenecientes a escuelas municipales de la comuna urbana de Hualpén, Región del Biobío, Chile.

\section{Material y Método}

\section{Diseño}

Considerando que los polimorfismos genéticos (Variables de exposición) son condiciones pre-existentes al estado nutricional (Variable de respuesta), nos pareció adecuado un diseño de casos y controles.

\section{Población de estudio}

Se reclutaron 241 niños obesos (casos) y 126 niños de peso normal (controles), con edades entre 7 y 11 años, residentes de la comuna urbana de Hualpén, Región del Biobío, Chile. Los sujetos fueron elegidos aleatoriamente a partir de un universo de 2.918 niños pertenecientes a escuelas municipales de Hualpén. Se excluyeron niños con diabetes mellitus tipo 1, disfunción tiroidea, disfunción suprarrenal u obesidad sindrómica. Los padres de los niños incluidos en este estudio firmaron un acta de consentimiento informado, de acuerdo a la declaración internacional de Helsinki sobre principios éticos para la investigación médica $^{23}$. El proyecto fue aprobado por el Comité de Ética de la Dirección de Investigación de la Universidad de Concepción.

\section{Antropometría y composición corporal}

El índice de masa corporal (IMC) fue expresado en $\left(\mathrm{kg} / \mathrm{m}^{2}\right)$. La talla fue medida a cada niño sin calzado, usando un estadiómetro marca Seca, modelo 208 con una precisión de $0,1 \mathrm{~cm}$. Para la medición del peso y la composición corporal se usó un impedanciómetro marca Tanita, modelo TBF-300, con una precisión de 0,1 kg. Los niños fueron clasificados como normopeso (IMC $\geq 5^{\text {th }}$ $\mathrm{y}<85^{\text {th }}$ percentil) $\mathrm{u}$ obeso $\left(\mathrm{IMC}>95^{\text {th }}\right.$ percentil) según la escala de percentiles definida por el $\mathrm{CDC}^{24}$ y de acuerdo a la Norma Técnica de Evaluación Nutricional de niños y niñas de 6 a 18 años del Ministerio de Salud ${ }^{25}$. Los niños fueron pesados en blusa o camisa, descalzos, sin portar objetos metálicos. La composición corporal fue medida con sistema de dos electrodos, pie a pie, con una frecuencia de $50 \mathrm{kHz}$, según las indicaciones del fabricante. El perímetro de la cintura fue medido pasando por borde superior de la cresta iliaca (a la altura del ombligo) con una cinta métrica no distensible, marca Seca, modelo 201, precisión de $0,1 \mathrm{~cm}$.

\section{Análisis de laboratorio \\ Metabolitos}

Los análisis de metabolitos circulantes fueron realizados a partir de una muestra de $4 \mathrm{ml}$ de sangre venosa tomada entre la 8.00 AM y 10.00 $\mathrm{AM}$, se exigió un ayuno mínimo de $4 \mathrm{~h}$, orinar 30 min antes del examen y no haber realizado ejercicio físico en las $24 \mathrm{~h}$ previas. Las mediciones de indicadores lipídicos y glucídicos circulantes fueron realizadas usando kits comerciales (Cobas C11Roche, Indianápolis IN, USA). La insulina y adiponectina fueron medidas utilizando kit de 
Polimorfismos del gen de adiponectina y estado nutricional en escolares - G. Orellana et al

ELISA comerciales (Linco Research, Missouri, USA) en un lector de placas de ELISA, Synergy 2 (Biotek, Vermont VT, USA). El índice HOMA-IR fue calculado a partir de las concentraciones basales de glucosa e insulina según ha sido previamente definido $^{26}$.

\section{Genotipificación}

Para la genotipificación de los polimorfismos del gen de la adiponectina se obtuvo ADN gnómico a partir de la fracción de leucocitos de sangre total utilizando un kit de extracción de genómico QIAamp DNA Blood Mini Kit (Quiagen $\mathrm{GmbH}$, Hilden, Germany) según el protocolo del fabricante. Los polimorfismos $+45 \mathrm{TG}$ y +276GT fueron determinados utilizando la técnica de reacción en cadena de la polimerasa (PCR) en tiempo real. La amplificación por PCR fue realizada en un volumen total de $25 \mathrm{ml}$ en un termociclador Rotor-Gene 6000 realtime PCR (Corbett Research, Sydney, Australia) y la mezcla de reacción contenía 100 ng de DNA genómico para SNP +276GT y 75 ng de DNA genómico para SNP +45TG. Además esta mezcla contenía 3,0 mM $\mathrm{MgCl}_{2}, 0,2 \mathrm{mM}$ dNTP, $200 \mathrm{nM}$ partidores, 1,5 mM tinción fluorescente Eva green, y 0,5 U Taq DNA polimerasa Platinum (Quantace, Taunton, USA). La amplificación se realizó usando partidores específicos para cada polimorfismos. Para SNP +45TG se usaron los partidores, sentido 5'-TGGACGGAGTCCTTTGTAGG-3' y antisentido 5'-TTGAGTCGTGGTTTCCTGGT-3'; y para SNP +276GT se usaron los partidores, sentido 5'-TCATCCTTGGAAGACCAAACC-3' y antisentido 5'-TTCTCACCCTTCTCACCAGG-3'. La reacción de amplificación fue llevada a cabo mediante el siguiente protocolo: $95^{\circ} \mathrm{C}$ por $10 \mathrm{~min}$, luego 40 ciclos de denaturación a $95^{\circ} \mathrm{C}$ por $15 \mathrm{~s}$, annealing por $10 \mathrm{~s}$ a $59^{\circ} \mathrm{C}$ y una extensión final a $72{ }^{\circ} \mathrm{C}$ por $10 \mathrm{~min}$. El análisis de HRM fue realizado entre $60^{\circ} \mathrm{C}$ y $90^{\circ} \mathrm{C}$, con una tasa de incrementos de temperatura de $0,1^{\circ} \mathrm{C} / \mathrm{s}$ para cada ensayo. Los datos de fluorescencia fueron adquiridos al final de cada etapa de annealing durante cada ciclo de PCR. Las curvas de HRM fueron realizadas usando el módulo del equipo. Cada genotipo fue identificado examinando gráficas de las curvas de melting diferenciales. Para este efecto se analizaron los tres genotipos posibles (ancestral, heterocigoto, homocigoto), previamente identificados mediante análisis de secuenciación. De esta forma se identificaron las curvas de melting con genotipo conocido (patrones) y las curvas problema fueron analizadas y comparadas en su similitud con las curvas patrones, aceptándose una similitud con 95\% de confianza o superior. Para confirmar la amplificación de un único producto $\mathrm{PCR}$, al final de la reacción, el producto fue analizado usando un gel de agarosa al 1\%. El tamaño de los productos esperados fueron de $161 \mathrm{pb}$ y $188 \mathrm{pb}$ para los SNP +45TG y +276GT respectivamente.

Los polimorfismos -11,377CG y -11,391GA ambos pertenecientes al promotor del gen fueron enviados a secuenciar al Departamento de Ecología, Facultad de Ciencias Biológicas, Pontificia Universidad Católica de Chile.

\section{Estadística}

Los análisis estadísticos fueron realizados con el Software estadístico SAS (SAS, versión 9.1-release Institute, Cary, NC). Previo a cada análisis los datos fueron sometidos a análisis de distribución usando el test Shapiro-Wilk. Las variables continuas fueron analizadas mediante test de t de Student. Si la variable no presentó una distribución normal la comparación entre grupos se realizó mediante el test de Mann-Whitney para dos grupos. Los resultados se expresaron para todos los analitos como promedio \pm desviación estándar y como mediana y sus respectivos rangos intercuartílicos. Las significancias estadísticas se calcularon de acuerdo al test indicado para cada tipo de distribución.

Las variables categóricas fueron representadas como porcentajes y analizadas mediante test de $\chi^{2}$ con 2 grados de libertad. Con la finalidad de confirmar si los genotipos se ajustaron al equilibrio de Hardy-Weinberg se aplicó el test de bondad de ajuste de $\chi^{2}$. La magnitud de la asociación de cada polimorfismo de AdipoQ con el estado nutricional (obesidad/no obesidad) fue estimada mediante regresión logística y expresada como OR. El error de muestreo de expresó como el intervalo de confianza (IC 95\%). La asociación entre los niveles de adiponectina y los polimorfismos genéticos de AdipoQ fue analizada mediante regresión lineal. Ajustes para potenciales variables confundentes fueron aplicadas cuando se consideró apropiado. Los valores fueron expresados como promedios \pm desviación estándar (DE) y para todos los análisis, el nivel de significación fue definido como $\mathrm{p}<0,05$. 


\section{Resultados}

\section{Caracterización de la población}

Las características antropométricas y bioquímicas de la muestra según sexo y condición nutricional se describen en la Tabla 1. El nivel sérico promedio de adiponectina y de colesterol HDL fueron significativamente menores en mujeres y varones obesos que en sus pares eutróficos. En contraste los niveles séricos de triglicéridos e insulina basal así como HOMA-IR y la razón Tg/HDL promedios fueron significativamente mayores en sujetos obesos que en eutróficos.

\section{Frecuencias alélicas}

Las frecuencias alélicas de los cuatro polimorfismos del gen de la adiponectina según estado nutricional se presentan en la Tabla 2. Las frecuencias alélicas de los polimorfismos $+45 \mathrm{TG}$, +276GT y -11,391GA no presentaron diferencias significativas entre los individuos normopeso y obesos. Sin embargo, el polimorfismo -11,377CG

Tabla 1. Características bioquímicas y antropométricas basales

\begin{tabular}{|c|c|c|c|c|c|c|c|c|c|}
\hline \multirow[t]{2}{*}{ Parámetro } & \multicolumn{4}{|c|}{ Normopeso } & \multicolumn{4}{|c|}{ Obeso } & \multirow[b]{2}{*}{$\mathbf{p}$} \\
\hline & \multicolumn{2}{|c|}{$\begin{array}{l}\text { Promedio } \\
\text { (DE) }\end{array}$} & \multicolumn{2}{|c|}{$\begin{array}{l}\text { Mediana } \\
\text { (Q1-Q3) }\end{array}$} & \multicolumn{2}{|c|}{$\begin{array}{l}\text { Promedio } \\
\text { (DE) }\end{array}$} & \multicolumn{2}{|c|}{$\begin{array}{l}\text { Mediana } \\
\text { (Q1-Q3) }\end{array}$} & \\
\hline \multicolumn{10}{|l|}{ Masculino } \\
\hline Edad (años) ${ }^{\dagger \dagger}$ & 8,5 & $(1,4)$ & 8,4 & $(7,2-9,7)$ & 8,6 & $(1,5)$ & 8,7 & $(7,4-9,7)$ & n.s. \\
\hline $\operatorname{IMC}\left(\mathrm{kg} / \mathrm{m}^{2}\right)^{\dagger \dagger}$ & 16,9 & (1) & 16,7 & $(16,2-17,5)$ & 23,8 & (3) & 23,4 & $(21,7-25,1)$ & $<0,0001$ \\
\hline Puntaje $\mathrm{z}-\mathrm{IMC}^{\dagger \dagger}$ & 0,4 & $(0,3)$ & 0,5 & $(0,2-0,7)$ & 2,1 & $(0,3)$ & 2,1 & $(1,9-2,3)$ & $<0,0001$ \\
\hline Circunferencia cintura $(\mathrm{cm})^{\dagger}$ & 59,4 & $(4,6)$ & 58,6 & $(55,5-62)$ & 77,8 & $(8,6)$ & 77 & $(72-81,8)$ & $<0,0001$ \\
\hline Porcentaje de grasa $(\%)^{\dagger \dagger}$ & 17,8 & $(3,2)$ & 18 & $(15,5-19,6)$ & 32,9 & $(6,6)$ & 32,5 & $(27,6-36,7)$ & $<0,0001$ \\
\hline Colesterol Total $\left(\mathrm{mg} / \mathrm{dL}^{-1}\right)^{\dagger+}$ & 181 & (38) & 174 & $(153-195)$ & 185 & (33) & 184 & $(163-208)$ & n.s. \\
\hline Colesterol-LDL $\left(\mathrm{mg} / \mathrm{dL}^{-1}\right)^{\dagger}$ & 108 & (29) & 110 & $(86-125)$ & 114 & $(27)$ & 114 & $(94-130)$ & n.s. \\
\hline Colesterol-HDL $\left(\mathrm{mg} / \mathrm{dL}^{-1}\right)^{\dagger}$ & 59 & (13) & 57 & $(52-67)$ & 50 & (10) & 50 & $(43-55)$ & $<0,0001$ \\
\hline Triglicéridos $\left(\mathrm{mg} / \mathrm{dL}^{-1}\right)^{\dagger \dagger}$ & 76 & $(27)$ & 70 & $(56-88)$ & 125 & (76) & 109 & $(72-154)$ & $<0,0001$ \\
\hline Glicemia basal $\left(\mathrm{mg} / \mathrm{dL}^{-1}\right)^{\dagger}$ & 90 & (8) & 90 & $(84-96)$ & 91 & (8) & 90 & $(84-97)$ & n.s. \\
\hline Insulina basal $\left(\mathrm{mU} / \mathrm{mL}^{-1}\right)^{\dagger \dagger}$ & 4,6 & $(3,2)$ & 3,8 & $(2,4-5,4)$ & 8,6 & $(4,9)$ & 7,6 & $(5,2-10,7)$ & $<0,0001$ \\
\hline $\mathrm{HOMA}-\mathrm{IR}^{+\dagger}$ & 1,1 & $(0,8)$ & 0,8 & $(0,5-1,3)$ & 1,9 & $(1,1)$ & 1,7 & $(1,2-2,4)$ & $<0,0001$ \\
\hline Adiponectina $\left(\mu \mathrm{g} / \mathrm{mL}^{-1}\right)^{\dagger+}$ & 17 & (7) & 15 & $(11-21)$ & 14 & (6) & 13 & $(10-16)$ & $<0,0001$ \\
\hline $\mathrm{Tg} / \mathrm{HDL}^{\dagger \dagger}$ & 1,4 & $(0,6)$ & 1,2 & $(1-1,5)$ & 2,8 & $(2,4)$ & 2,1 & $(1,3-3,5)$ & 0,0006 \\
\hline \multicolumn{10}{|l|}{ Femenino } \\
\hline Edad (años) ${ }^{\dagger+}$ & 8,5 & $(1,5)$ & 8,5 & $(7,1-9,6)$ & 8,5 & $(1,4)$ & 8,6 & $(7,2-9,7)$ & n.s. \\
\hline IMC $\left(\mathrm{kg} / \mathrm{m}^{2}\right)^{\dagger+}$ & 17,2 & $(1,2)$ & 17,1 & $(16,3-18)$ & 24 & $(2,7)$ & 23,8 & $(22,2-25,3)$ & $<0,0001$ \\
\hline Puntaje $\mathrm{z}-\mathrm{IMC}^{\dagger \dagger}$ & 0,5 & $(0,4)$ & 0,5 & $(0,3-0,7)$ & 2 & $(0,3)$ & 2 & $(1,8-2,2)$ & $<0,0001$ \\
\hline Circunferencia cintura $(\mathrm{cm})^{\dagger \dagger}$ & 62 & $(6,3)$ & 61,5 & $(56,1-67)$ & 78,8 & $(8,7)$ & 79 & $(72-84)$ & $<0,0001$ \\
\hline Porcentaje de grasa $(\%)^{\dagger}$ & 21,1 & $(5,9)$ & 21,2 & $(16,9-24,6)$ & 36,4 & $(4,6)$ & 36,5 & $(33,4-38,9)$ & $<0,0001$ \\
\hline Colesterol Total $\left(\mathrm{mg} / \mathrm{dL}^{-1}\right)^{\dagger+}$ & 182 & (34) & 177 & $(159-198)$ & 184 & (37) & 178 & $(160-205)$ & n.s. \\
\hline Colesterol LDL $\left(\mathrm{mg} / \mathrm{dL}^{-1}\right)^{\dagger+}$ & 106 & (27) & 105 & $(83-124)$ & 109 & (31) & 104 & $(87-122)$ & n.s. \\
\hline Colesterol HDL $\left(\mathrm{mg} / \mathrm{dL}^{-1}\right)^{\dagger}$ & 56 & (13) & 56 & $(47-64)$ & 47 & (11) & 46 & $(39-55)$ & $<0,0001$ \\
\hline Triglicéridos $\left(\mathrm{mg} / \mathrm{dL}^{-1}\right)^{\dagger+}$ & 101 & $(48)$ & 92 & $(63-128)$ & 139 & (73) & 123 & $(85-175)$ & $<0,0001$ \\
\hline Glicemia $\left(\mathrm{mg} / \mathrm{dL}^{-1}\right)^{\dagger}$ & 86 & (7) & 86 & $(82-91)$ & 87 & (10) & 87 & $(80-92)$ & n.s. \\
\hline Insulina $\left(\mathrm{mU} / \mathrm{mL}^{-1}\right)^{\text {t+ }}$ & 5,7 & $(4,5)$ & 4,5 & $(2,7-6,7)$ & 10,5 & $(8,3)$ & 8,4 & $(5,1-13,5)$ & $<0,0001$ \\
\hline HOMA-IR ${ }^{+\dagger}$ & 1,2 & (1) & 0,9 & $(0,6-1,4)$ & 2,2 & $(1,6)$ & 1,8 & $(1,1-2,9)$ & $<0,0001$ \\
\hline Adiponectina $\left(\mu \mathrm{g} / \mathrm{mL}^{-1}\right)^{\dagger+}$ & 17 & (6) & 16 & $(13-21)$ & 13 & (5) & 13 & $(10-15)$ & $<0,0001$ \\
\hline $\mathrm{Tg} / \mathrm{HDL}^{+\dagger}$ & 2 & $(1,3)$ & 1,7 & $(1,1-2,3)$ & 3,4 & $(2,7)$ & 2,7 & $(1,7-4,4)$ & $<0,0001$ \\
\hline
\end{tabular}

DE: Desviación Estándar, $\mathrm{Q}_{1}-\mathrm{Q}_{3}$ : Quartil 1 - Quartil 3. 'test t de Student. ${ }^{\dagger}$ test Mann-Whitney. 
Polimorfismos del gen de adiponectina y estado nutricional en escolares - G. Orellana et al

Tabla 2. Frecuencias de los genotipos, según estado nutricional

\begin{tabular}{|c|c|c|c|c|c|c|}
\hline & & $\begin{array}{c}\text { Ancestral } \\
\% \quad \text { (n) }\end{array}$ & $\begin{array}{c}\text { Heter } \\
\%\end{array}$ & $\begin{array}{l}\text { cigoto } \\
\text { (n) }\end{array}$ & $\begin{array}{c}\text { Homocigoto } \\
\% \quad \text { (n) }\end{array}$ & $\mathbf{p}$ \\
\hline \multirow[t]{2}{*}{$+45 \mathrm{TG}$} & Normopeso & $69 \quad(88)$ & 31 & (39) & (0) & 0,458 \\
\hline & Obeso & $75 \quad(178)$ & 26 & (62) & (1) & \\
\hline \multirow[t]{2}{*}{$+276 \mathrm{GT}$} & Normopeso & $67 \quad(85)$ & 18 & (23) & 15 (19) & 0,742 \\
\hline & Obeso & $66 \quad(159)$ & 16 & (39) & 18 (43) & \\
\hline \multirow[t]{2}{*}{$-11,377 C G$} & Normopeso & $76 \quad(97)$ & 20 & (25) & $4 \quad(5)$ & $<0,0001$ \\
\hline & Obeso & $75(181)$ & 6 & (15) & 19 (45) & \\
\hline \multirow[t]{2}{*}{$-11,391 \mathrm{GA}$} & Normopeso & $72 \quad(92)$ & 27 & (34) & (1) & 0,420 \\
\hline & Obeso & 78 (189) & 21 & $(51)$ & (1) & \\
\hline
\end{tabular}

mostró una frecuencia alélica del alelo G (alelo menor), que resultó ser significativamente superior en niños obesos de ambos sexos, comparados con los de peso normal $(\mathrm{p}<0,0001)$.

\section{Asociación entre polimorfismos del gen} de la adiponectina y obesidad

Con la finalidad de estimar el riesgo de obesidad para cada polimorfismo, se confirmó el cumplimiento del equilibrio de Hardy-Weinberg. Posteriormente, se calcularon los ORs (IC 95\%) según los modelos de herencia codominante, recesivo y aditivo. Este análisis para el polimorfismo -11,377CC se muestra en la Tabla 3, donde se aprecia que la presencia de este polimorfismo se asocia con un mayor riesgo de obesidad. No se encontró asociación entre las otras variantes genéticas analizadas y el estado nutricional.

\section{Asociación entre la concentración de} adiponectina plasmática y polimorfismos del gen

La Figura 1 muestra la asociación entre la concentración plasmática de adiponectina y los polimorfismos del gen para cada estado nutricional. En la mayoría de los casos la obesidad se asoció a una reducción significativa de las concentraciones de adiponectina. En el caso de polimorfismos +276GT, se observa que la adición de
Tabla 3. Análisis del polimorfismo -11,377CG del gen de la adiponectina en relación a la obesidad con diferentes modelos de herencia

\begin{tabular}{|c|c|c|c|c|}
\hline Modelo & Genotipo & OR & $(95 \% \mathrm{Cl})$ & $\mathbf{p}$ \\
\hline \multirow[t]{3}{*}{ Codominante } & CC & 1,00 & & \\
\hline & CG & 0,297 & $(0,148-0,598)$ & 0,0007 \\
\hline & GG & 4,773 & $(1,834-12,423)$ & 0,0014 \\
\hline \multirow[t]{2}{*}{ Recesivo } & CC-CG & 1,00 & & \\
\hline & GG & 5,585 & $(2,157-14,460)$ & 0,0004 \\
\hline \multirow[t]{2}{*}{ Aditivo } & CC & 1,00 & & \\
\hline & CC-CG- GG & 1,389 & $(1,001-1,929)$ & 0,0496 \\
\hline
\end{tabular}

un alelo de riesgo se asocia con reducción de los niveles circulantes de adiponectina, sin embargo, tal reducción no alcanza significancia estadística dentro de un mismo estado nutricional. En el caso del polimorfismo -11,377CG ocurre algo parecido pero sólo en condición de obesidad. Las variantes genéticas $+45 \mathrm{TG}$ y -11,391GA no modifican el efecto de la condición de obesidad sobre el nivel sérico de adiponectina.

\section{Discusión}

La obesidad infantil se asocia a un elevado riesgo de perpetuar la condición de obesidad en la vida adulta y constituye un factor de riesgo independiente para el desarrollo de trastornos metabólicos como resistencia a la insulina y dislipidemias o enfermedades como hipertensión arterial, DM2 y eventos cardiovasculares ${ }^{1}$. 


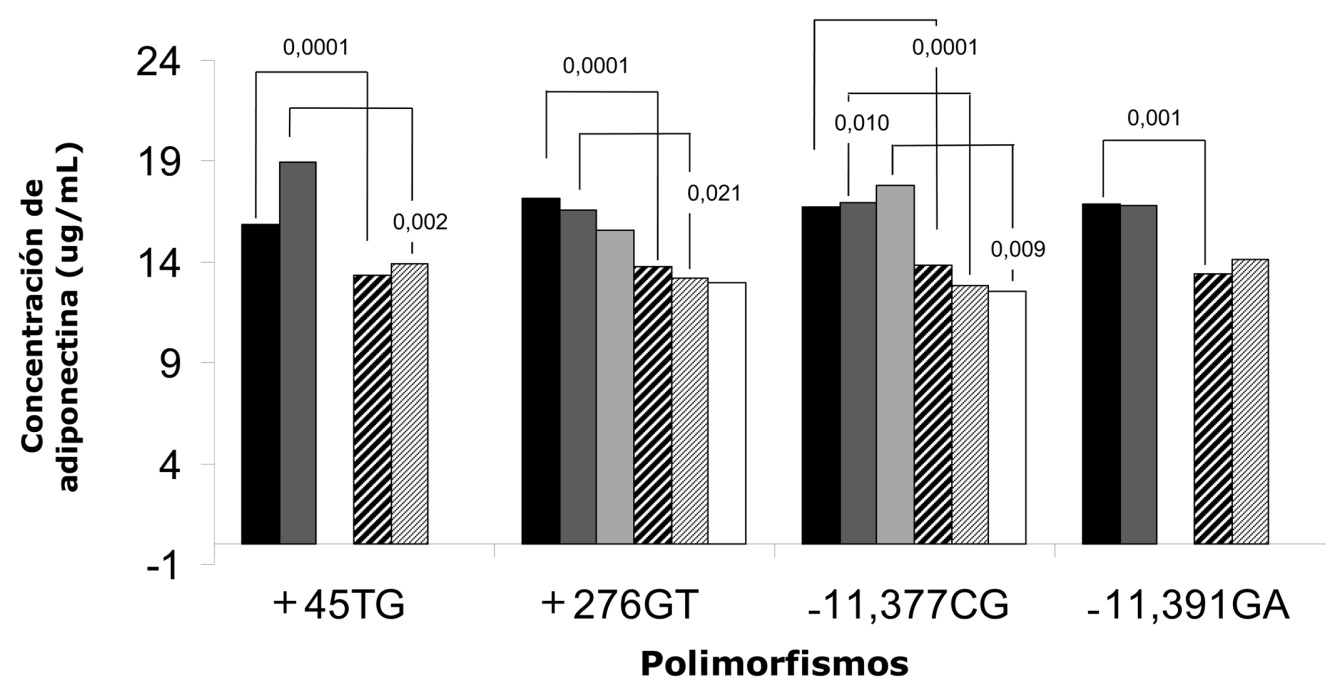

NP Ancestral

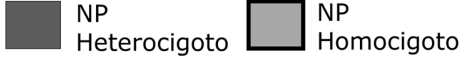

Ob Ancestral

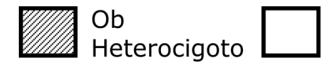

$\mathrm{Ob}$ Homocigoto

Figura 1. Concentraciones plasmáticas de adiponectina, según polimorfismo genético (ancestral, heterocigoto u homocigoto para la variante genética respectiva) y estado nutricional de los escolares (NP = normopeso y Ob = obeso). Las diferencia estadísticamente significativas se señalan con la siguiente simbología: ${ }^{*} p<0,05,{ }^{* *} p<0,01$ y *** $p<0,001$.

El tejido adiposo es un importante órgano endocrino que secreta hormonas conocidas como adipoquinas $^{9}$. La adiponectina es la adipoquina secretada en mayor cantidad por el tejido adipo$\mathrm{so}^{4,8}$. Al igual que otros estudios ${ }^{4,14}$, encontramos que la concentración sérica de adiponectina es significativamente inferior en niños obesos de ambos sexos. Nuestros resultados sugieren que dos variantes genéticas del gen de la adiponectina (+276GT y -11,377CG) potenciarían la reducción de la concentración sérica de adiponectina relacionada con el peso, aunque sin alcanzar significación estadística. El hecho de que otros autores hayan demostrado que las variantes +276GT y -11,377CG se asocian con una menor concentración sérica de adiponectina ${ }^{17,20}$ podría atribuirse a un mayor tamaño muestral o una edad más avanzada de la muestra.

El mecanismo por el cual el polimorfismo -11,377CG, ubicado en la región promotora del gen, se asocia con menor concentración sérica de adiponectina debería estar relacionado con la menor actividad transcripcional del promotor que contiene la variante genética, según ha sido reportado por otros autores ${ }^{12,14,28}$.

En el presente estudio, realizado en niños de Hualpén, región del Biobío, se comunica por primera vez la asociación entre el polimorfismo -11.377CG con obesidad, en población chilena. La frecuencia del polimorfismo fue 38,8\% mayor en niños obesos que en eutróficos (OR 1,389 IC $95 \% 1,001-1,929 \mathrm{p}=0,0496)$ según el modelo aditivo. Bouatia-Naji et al. ${ }^{14}$ determinaron que el polimorfismo -11,377CG era $24 \%$ más frecuente en niños franceses con obesidad extrema (OR 1,24 IC 95\% 1,03-1,50 p = 0,025) según el modelo dominante. Los OR calculados por los modelos codominante y recesivo fueron de 4,773 (IC 95\% 1,834-12,423 p = 0,0007) y 5,585 (IC $95 \%$ 2,157-14,460 $\mathrm{p}=0,0004)$ respectivamente. Este resultado es relevante ya que la etiología de la obesidad es multifactorial y la expresión del fenotipo obeso responde a complejas interacciones entre genes y medio ambiente. Recientemente, el consorcio GWAS identificó 8 loci con mutaciones puntuales asociadas ampliamente con obesidad, 
Polimorfismos del gen de adiponectina y estado nutricional en escolares - G. Orellana et al

sin embargo, ninguno de estos locus corresponde al gen de la adiponectina ${ }^{27}$. Esto puede significar que la asociación del polimorfismo -11.377CG con obesidad está restringida sólo a determinadas poblaciones, entre ellas la población chilena estudiada, con una carga genética predominantemente amerindia, como lo sugieren algunos estudios ${ }^{29,30}$. El significado metabólico expresado en niveles de insulina plasmática no manifestó diferencias entre ambos grupos (resultados no mostrados), sin embargo, hay que considerar que la población analizada tiene una edad promedio de alrededor de 8,5 años, edad en la cual pudieran existir otros mecanismos de compensación y por otra parte, variables confundentes como el estado puberal heterogéneo, entre otras.

Aunque no encontramos que los polimorfismos +45TG, +276GT y -11,391GA se asocien a un mayor riesgo de obesidad, la frecuencia de los alelos menores de esta variantes fue considerable ( $\geq 11 \%$ ) (Tabla 2). Otros autores han demostrado frecuencia alélicas similares para los polimorfismo -11,394, -11,391GA, +276GT y +45TG en niños franceses y polacos caucásicos ${ }^{14,22}$.

El hecho de que los polimorfismos 45TG, +276GT sean prevalentes en nuestra población adquiere relevancia si se considera que muchos estudios han revelado asociación entre estos polimorfismos y un mayor riesgo de insulino resistencia y DM2 $2^{11,13,17,18,19}$.

El pequeño tamaño de la muestra estudiada limita el poder estadístico de nuestro estudio. Por otra parte, sólo se analizaron dos polimorfismos de la región promotora $(-11391 \mathrm{~A} / \mathrm{G}$ y $-11377 \mathrm{C} / \mathrm{G})$, omitiendo el polimorfismo $-11426 \mathrm{~A} / \mathrm{G}$ que se expresa con una frecuencia mayor que $1,5 \%{ }^{12}$.

En conclusión, esta investigación demostró que niños escolares obesos de la Región del Biobío, tienen un riesgo aumentado de portar el alelo de susceptibilidad del polimorfismo $-11377 \mathrm{C} / \mathrm{G}$ del gen de la adiponectina. También se concluye que existe una importante frecuencia alélica de los polimorfismos $+45 \mathrm{TG},+276 \mathrm{GT}$, que se han asociado en múltiples estudios con mayor riesgo de diabetes mellitus 2 y eventos cardiovasculares.

\section{Referencias}

1. Hills A, Okely A, Baur L. Addressing childhood obesity through increased physical activity. Nat Rev Endocrinol 2010; 6: 543-9.
2. Bustos P, Sáez K, Gleisner A, Ulloa N, Calvo C, Asenjo S. Metabolic syndrome in obese adolescents. Pediatr Diabetes 2010; 11: 55-60.

3. Departamento de estadística e Información de Salud (DEIS). Diagnóstico nutricional integrado de la población menor de 6 años, por regiones, 2011. Ministerio de Salud. Disponible en http://deis.minsal.cl/index.asp

4. Arnaiz H, Acevedo M, Barja S, Aglony M, Guzmán B, Cassis B, et al. Adiponectin levels, cardiometabolic risk factors and markers of subclinical atherosclerosis in children. Int J Cardiol 2010; 138 (2): 138-44.

5. Chiarelli F, Marcovecchio M. Insulin resistance and obesity in childhood. Eur J Endocrinol 2008; 159: S67-S74.

6. Lee YS. Consequences of childhood obesity. Ann Acad Med Singapore 2009; 38: 75-81.

7. Benjamin S, Cradock A, Walker E, Slining M, Gillman M. Obesity prevention in child care: a review of U.S. state regulations. BMC Publich Health 2008; 8: 188.

8. Liu M, Liu F. Transcriptional and post-translational regulation of adiponectin. Biochem J 2010; 425: 41-52

9. Brochu-Gaudreau K, Rehfeldt C, Blouin R, Bordignon V, Murphy BD, Palin MF. Adiponectin action from head to toe. Endocrina 2010; 37 (1): 11-32. Epub 2009 Dec 1.

10. Capeau J. The story of the adiponectin and its receptors AdipoR1 and AdipoR2: To follow. J Hepatol 2007; 47: 736-8.

11. Kyriakou T, Collins L, Spencer-Jones N, Malcolm C, Wang X, Snieder H, et al. Adiponectin gene ADIPOQ SNP associations with serum adiponectin in two female populations and effects of SNPs on promoter activity. J Hum Genet 2008; 53 (8): 718-27.

12. Gu Harvest. Biomarkers of adiponectina: Plasma proteín variation and genomic DNA polymorphisms. Biomark Insights 2009; 4: 23-133.

13. Sun H, Gong Z, Yin J, Liu H, Liu Y, Guo Z, et al. The association of adiponectin allele $45 \mathrm{~T} / \mathrm{G}$ and $-11377 \mathrm{C} / \mathrm{G}$ polymorphisms with type 2 diabetes and rosiglitazone response in chinese patients. Br J Pharmacol 2008; 65 (6): 917-26.

14. Bouatia-Naji N, Meyre D, Lobbens S, Seron K, Fumeron F, Balkau B, et al. ACDC/adiponectin polymorphisms are associated with severe childhood and adult obesity. Diabetes 2006; 55: 545-50.

15. Gable DR, Hurel SJ, Humphries SE. Adiponectin and its gene variants as risk factors for insuline resistance, the metabolic syndrome and cardiovascular disease. Atherosclerosis 2006; 188 (2): 231-44.

16. Melistas L, Mantzoros C, Kontogianni M, Antonopoulou $\mathrm{S}$, Ordovas J, Yiannakouris N. Association of the $+45 \mathrm{~T}$ $>\mathrm{G}$ and $+276 \mathrm{G}>\mathrm{T}$ polymorphisms in the adiponectin gene with insulin resistance in nondiabetic Greek wo- 
Polimorfismos del gen de adiponectina y estado nutricional en escolares - G. Orellana et al

men. Europ J Endocrinol 2009; 161: 845-52.

17. Pérez-Martines P, López-Miranda J, Cruz-Teno C, Delgado-Lista J, Jiménez-Gómez Y, Fernández J, et al. Adiponectin gene variants are associated with insulin sensitivity in response to dietary fat consumption in caucasian men. J Nutr 2008; 138: 1609-14.

18. Verduci $\mathrm{E}$ Scaglioni \$ Agostoni O Radaelli G, Biondi M Manso AS, et al. The relationship of insulin resistance with SNP 276G > T at adiponectin gene and plasma long-chain polyunsaturated fatty acids in obese children. Pediatr Res 2009; 66 (3): 346-9.

19. Petrone A, Zavarella S, Caiazzo A, Leto G, Spoletini M, Potenziani S, et al. The Promoter region of the adiponectin gene is a determinant in modulating insulin sensitivity in childhood obesity. Obesity 2006; 14: 1498-504.

20. Buzzetti R, Petrone A, Zavarella S, Zampetti S, Spoletini $\mathrm{M}$, Potenzian S, et al. The glucose clamp reveals an association between adiponectin gene polymorphisms and insulin sensitivity in obese subjects. Int J Obes 2007; 31: 424-8.

21. Tanko L, Siddiq A, Lecoeur C, Larsen P, Christiansen C, Walley A, et al. ACDC/Adiponectin and PPAR- gene polymorphisms: implications for features of obesity. Obesity 2005; 13 (12): 2113-21.

22. Szopa M, Malczewska-Malec M, Wilk B, Skupien J, Wolkow P, Malecki M, et al. Variants of the adiponectin gene and type 2 diabetes in a polish population. Acta Diabetol 2008; 46 (4): 317-22.

23. Human Experimentation: Code of Ethics of the World Medical Association (Declaration of Helsinki), Can Med Assoc J 1964; 91 (11): 619.
24. Flegal K, Tabak C, Ogden C. Overweight in children: definitions and interpretations. Health Educ Res 2006; 21 (6): 755-60.

25. Ministerio de Salud de Chile. Disponible en Www.minsal.c

26. Matthews DR, Hosker JP, Rudenski AS, Naylor BA, Treacher DF, Turner RC. Homeostasis model assessment: insulin resistance and beta-cell function from fasting plasma glucose and insulin concentrations in man. Diabetologia 1985: 28; 412-9.

27. Willer C), Speliotes EK, Loos R, Li \$, Lindgren CM, Heid IM, et al. Six new loci associated with body mass index highlight a neuronal influence on body weight regulation. Nat Genet 2009; 41 (1): 25-34. Epub 2008 Dec 14.

28. Vasseur F, Helbecque N, Lobbens S, Vasseur-Delannoy V, Dina C, Clèment $\mathrm{K}$, et al. Hypoadiponectinemia and high risk of type 2 diabetes are associated with adiponectin-enconding (ACDC) gene promoter variants in morbid obesity: evidence for a role of ACDC in diabesity. Diabetología 2005; 48: 892-9.

29. Moraga ML, Rocco P, Miquel JF, Nervi F, Llop E, Chakraborty $\mathrm{R}$, et al. Mitochondrial DNA polymorphisms in Chilean aboriginal populations: implications for the peopling of the southern cone of the continent. Am J Phys Anthropol 2000; 113: 19-29.

30. Guzmán N, Lanas F, Salazar LA. Influence of Amerindian mitochondrial DNA haplogroups on thrombosis susceptibility and frequency of four genetic prothrombotic variants in Southern Chilean subjects. Clin Chim Acta 2010; 411: 444-7. 